\title{
Polar domains in lead titanate films under tensile strain
}

\author{
G. Catalan ${ }^{1,3},{ }^{*}$ A. Janssens ${ }^{2}$, G. Rispens ${ }^{1}$, S. Csiszar ${ }^{1}$, O. Seeck ${ }^{4}$, G. Rijnders ${ }^{2}$, D.H.A. Blank ${ }^{2}$, and B. Noheda ${ }^{1 \dagger}$ \\ ${ }^{1}$ Materials Science Centre, University of Groningen, Groningen 9747AG, The Netherlands \\ ${ }^{2}$ MESA + Institute for Nanotechnology, Twente University, Enschede 7500AE, The Netherlands \\ ${ }^{3}$ Department of Earth Sciences, University of Cambridge, Cambridge CB2 3EQ, United Kingdom and \\ ${ }^{4}$ HASYLAB- DESY, Notkestr. 85, D-22603 Hamburg, Germany
}

(Dated: January 22, 2006 (revised version))

\begin{abstract}
Thin films of $\mathrm{PbTiO}_{3}$, a classical ferroelectric, have been grown under tensile strain on singlecrystal substrates of $\mathrm{DyScO}_{3}$. The films, of only $5 \mathrm{~nm}$ thickness, grow fully coherent with the substrate and show no crystallographic twin domains, as evidenced by synchrotron x-ray diffraction. A mapping of the reciprocal space reveals intensity modulations (satellites) due to regularly-spaced polar domains in which the polarization appears rotated away from the substrate normal, characterizing a low symmetry phase not observed in the bulk material. This could have important practical implications since these phases are known to be responsible for ultrahigh piezoelectric responses in complex systems.
\end{abstract}

PACS numbers: 77.55.+f, 77.80.-e, 68.55.-a, 61.10.-i

Ferroelectrics are dielectric materials with a permanent switchable polarization. Ferroelectricity as a critical phenomenon is of broad fundamental interest. Theoretical and experimental research of ferroelectric thin films is recently attracting a lot of attention not only because of their promising technological applications in novel geometries but also because of the new fundamental understanding of ferroelectricity at the nanoscale[1].

Perovskite ferroelectric thin films are strongly affected by epitaxial strain. Clamping between the film and the substrate onto which it is deposited is known to induce shifts in critical temperatures, increase tetragonality and polarisation, and change the order of the phase transition [2-7]. Some theoretical works have also predicted other interesting possibilities, such as changing the polar symmetry [7-9], engineering the domain configuration $[10,11]$ and, for unscreened surface charges, inducing polar rotations across the thickness of the films [12].

The most interesting predictions of the stability diagrams for epitaxial tetragonal perovskite ferroelectrics tend to be those for tensile in-plane strain [7-11], as compressive strains simply induce an enhancement of tetragonal out-of-plane polarization. However, experimental verification of such predictions is difficult. This is partly because most available perovskite single-crystal substrates $\left(\mathrm{SrTiO}_{3}, \mathrm{LaAlO}_{3}, \mathrm{NdGaO}_{3}\right)$ have lattice parameters that are either very close to, or smaller than, those of typical perovskite ferroelectrics such as $\mathrm{PbTiO}_{3}$ or $\mathrm{BaTiO}_{3}$. At the other end of the spectrum, substrates with bigger lattice parameters, such as perovskite $\mathrm{KTaO}_{3}$, have so large a mismatch that the strain is rapidly relaxed through dislocations or twin formation[13, 14]. Fortunately, the recent development of new perovskite single crystals from the family of the

\footnotetext{
*Electronic address: gcat05@esc.cam.ac.uk

${ }^{\dagger}$ Electronic address: b.noheda@rug.nl; Corresponding author
}

scandates $[3,4,15]$ bridges the gap, thereby widening the scope for strain tailoring of the films and allowing us to explore experimentally some of the above-mentioned theoretical predictions.

We have grown fully coherent thin films of the archetypal perovskite ferroelectric $\mathrm{PbTiO}_{3}$ (PTO) under biaxial tensile strain and analyzed the reciprocal space using synchrotron x-ray diffraction. The results show that polarization rotation is induced in these films. This new polar state of PTO is consistent with the so-called $a c$ -polarization tilted in the (010) plane- and $r$-polarization tilted in the (110) plane- phases. Neither symmetry exists in the parent bulk compound [16]. These findings could have important technological implications since similar low symmetry phases, very rare in ferroelectrics, are believed to be responsible for the unusually large responses observed in the ultrahigh piezoelectrics, such as $\mathrm{PbZr}_{1-x} \mathrm{Ti}_{x} \mathrm{O}_{3}$ and related systems[17, 18]. The results thus confirm experimentally that new polar states that do not exist in bulk can be made accessible in epitaxial films through careful tuning of strain, thickness and electrode [7, 8].

Experimental studies of ferroelectricity often require either the use of electrodes, which themselves introduce important changes in the thin films $[12,19]$, or optical methods, unfeasible when the optical thickness of the films is smaller than the wavelength. However, in a series of seminal papers, Streiffer and co-workers have shown that polar stripe domains can be detected as satellite peaks in x-ray diffraction patterns, and that this technique can be used to measure the domains size and polar orientation [20,21] even in films as thin as 3 perovskite unit cells [21]. This technique is therefore ideally suited for the study of polar symmetry in very thin films where very few others (see ref. [22]) can be implemented.

In this study we have deposited $5 \mathrm{~nm}$ thin films of PTO, using RHEED-assisted Pulsed Laser Deposition, at $570^{\circ} \mathrm{C}$ and with an oxygen background pressure of 0.13 mbar (further details of the growth will be pub- 


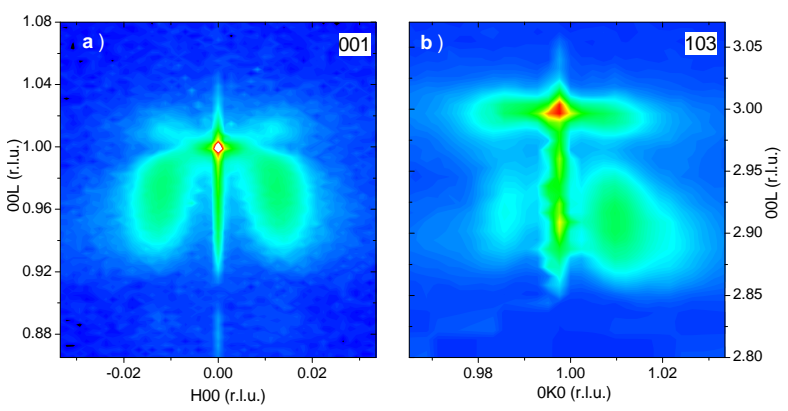

FIG. 1: (Color online)Logarithmic reciprocal maps in the H0L scattering plane around the 001 (a) and 103 (b) reflections for a $5 \mathrm{~nm} \mathrm{PbTiO}_{3}$ film directly grown on a $\mathrm{DyScO}_{3}$ substrate. (Intensities from low to high: blue-green-yellow-red-white)

lished elsewhere[23]). The films were grown either directly on [110]-DSO crystals, or on [110]-DSO with a $5 \mathrm{~nm}$ buffer layer of the conductive perovskite $\mathrm{SrRuO}_{3}$ (SRO), deposited at $600^{\circ} \mathrm{C}[24]$. Bulk PTO has a tetragonal structure at room temperature, with lattice parameters $\mathrm{a}=\mathrm{b}=3.89 \AA$ and $\mathrm{c}=4.14 \AA[25]$. The lattice structure of $\mathrm{DyScO}_{3}$ (DSO) is orthorhombic and [110]-oriented substrates are expected to have nearly-squared in-plane lattice with $\mathrm{a}=3.944-3.948 \AA$ and $\mathrm{b}=3.943-3.945 \AA$ at right angles[4, 15], which would induce tensile strain $\left(\mathrm{a}_{\text {film }} / \mathrm{a}_{\text {substrate }}-1\right)$ of about $1.4 \%$ on the PTO film. The cubic structure of PTO around $T_{C}=490{ }^{\circ} \mathrm{C}$, has a lattice parameter that is almost identical to the pseudocubic lattice parameter of DSO [15] and at the deposition temperature there is good lattice match between PTO and DSO, while the mismatch between SRO and DSO is only $\approx 0.4$ [15]; this, in combination with the reduced thickness, prevents the relaxation of either layer via misfit dislocations [26]. As it cools down below $T_{C}$, however, the drive towards the tetragonal structure introduces large in-plane strains. These may be accommodated by dislocations [26], by domain twinning $[10,11,13]$ and/or by changes in the symmetry of the films $[7,8]$.

The synchrotron x-ray measurements were carried out in the W1 beamline at HASYLAB (DESY-Hamburg), using a $1 \times 1 \mathrm{~mm}^{2}$ monochromatic beam of $9.8 \mathrm{keV}(\lambda$ $=1.26515 \AA$ ). Reciprocal space maps within the $H 0 L$ and $0 K L$ zones were obtained in standard reflection geometry. Scans in the $H K 0$ zone were achieved using grazing incidence diffraction (GID), in which a non-propagating evanescent wave is used to diffract within the plane of the film. This is attained by making the $\mathrm{x}$-ray beam reach the surface close to the critical angle of total reflection $\left(0.3^{\circ}\right.$ in our case). The reciprocal space maps presented here have been calculated from the measured angular scans and plotted in reciprocal lattice units (r.l.u.) of the substrate $(1$ r.l.u. $=2 \pi / 3.950 \AA$, in-plane, and $=2 \pi / 3.942 \AA$, out-of-plane).

The reciprocal space maps around the (001) and (103) reflections (Figure 1) show that the in-plane reciprocal space vectors of the film and the substrate (the sharp
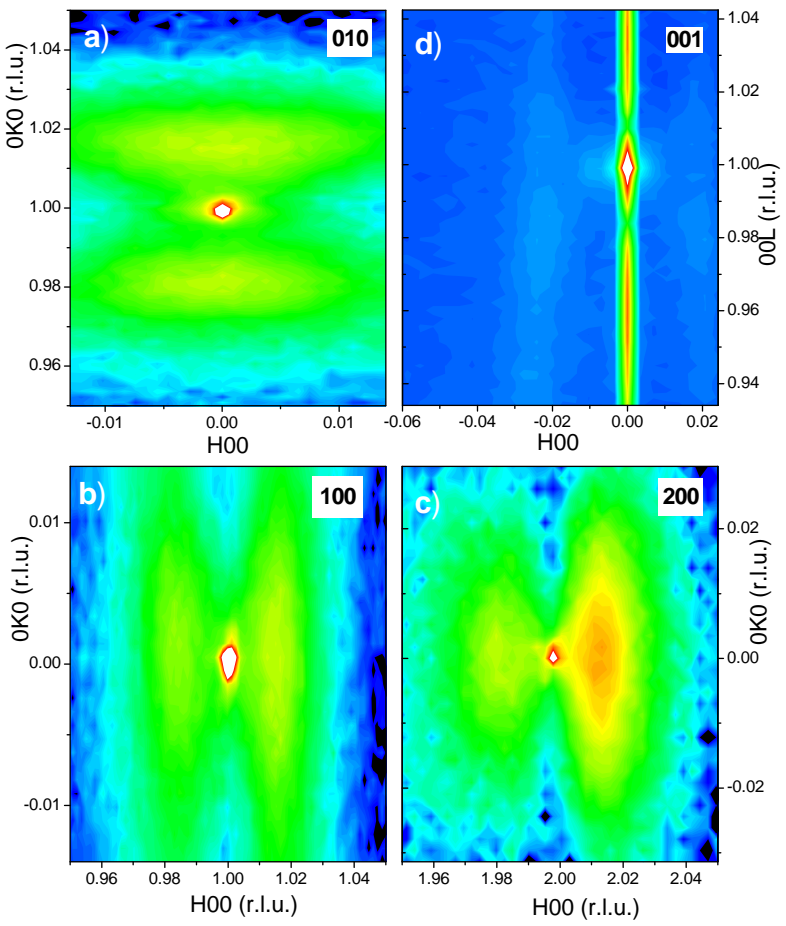

FIG. 2: (Color online) Logarithmic reciprocal space maps in the HK0 scattering zone around the a) 010, b)100 and c)200 reflections; and in the H0L zone around the 001 reflection (d), for the film grown with an SRO electrode. (Intensities from low to high: b-g-y-r-w)

peaks at $\mathrm{L}=1$ (a) and $\mathrm{L}=3(\mathrm{~b})$ ) are identical (same $\mathrm{H}$ value in the figures), and that the films' truncation rods have the same width as the substrate peak. These observations are evidence of full in-plane coherence of the films. However, Figure 1 also shows broad satellite peaks outside of the truncation rod. In Figure 2 the GID reciprocal space maps within the HK0 plane, corresponding to the (100), (010) and (200) in-plane reflections of the film grown with a SRO buffer layer are shown, together with the scan in the H0L plane around the specular 001 reflection of the same sample. For a given sample, the satellites have the same reciprocal space separation in all reflections. This shows that they are not Bragg peaks owing to different elastic domains, or twins, but originate instead from an in-plane modulation. The distance between the satellites and the Bragg peak $(\Delta H)$ yields modulation periodicities of $\Lambda=2 \pi / \Delta Q=0.395 \mathrm{~nm} / \Delta H \simeq 20 \mathrm{~nm}$ for the films grown with an SRO electrode, and $\Lambda \simeq 30 \mathrm{~nm}$ for the films grown directly on DSO.

Figure 3 shows the HKO area scans around the 110 reflections of the two films. While the satellites for the film grown on an SRO electrode are oriented preferentially along the a, b pseudo-cubic axes (the edges of the substrate), the intensity distribution for the film grown directly on DSO is somewhat more isotropic (same nearcircular distribution with increased intensity at high $\mathrm{H}$ and $\mathrm{K}$ values was found around the 100 and 010 reflec- 

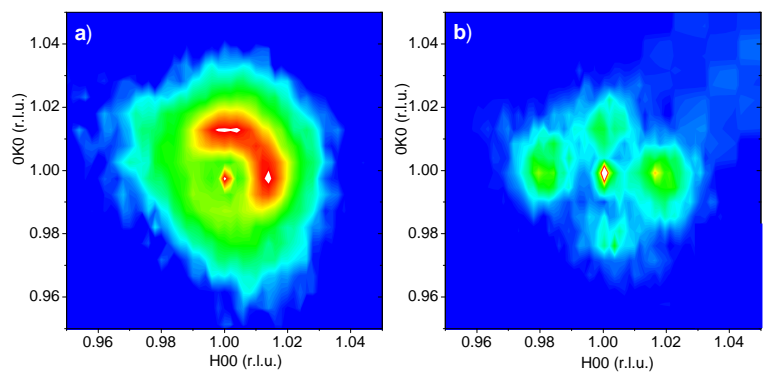

FIG. 3: (Color online)Logarithmic reciprocal space maps of the HK0 plane around the 110 Bragg peaks of the films grown a)directly on DSO, and b)on DSO with an SRO bottom electrode. (Intensities from low to high: b-g-y-r-w)

tions of the same sample). We do not know the cause for this difference, although we note that both types of domain configuration have been seen before for PTO films grown on $\mathrm{SrTiO}_{3}$ substrates[20, 21].

The pseudocubic lattice parameters of the DSO substrate were found to be $a \simeq b=3.950 \AA$, and $c=3.942$ $\AA$, while the lattice parameters of the PTO films were $\mathrm{a} \simeq \mathrm{b}=3.950 \AA$, owing to the in-plane coherence, and $\mathrm{c} \simeq 4.07 \AA[27]$. As reported by He et al. [5, 28], the clamping to the substrate can decouple the symmetry of the unit cell from that of the polar shifts. Moreover, from a functional point of view, as important as the crystal structure is the polar symmetry and its domain configuration. In order to elucidate this, we turn to the analysis of the satellite peaks.

In-plane satellites in x-ray diffraction patterns may have different origins: grain boundaries, dislocations, disclinations, or other periodic structural discontinuities, or else, in ferroelectric materials, polar domains. We can disregard grain boundaries from AFM analysis of the film surface [23]. As for misfit dislocations, the good lattice match during growth makes them unlikely. In fact, the symmetry of the 100 and 010 satellites, along [h00] and [0k0], respectively (Figure 2) is inconsistent with scattering by dislocations, grain boundaries or any other periodic defects, since they should render modulation along the same directions in all reflections, which is not the case. Thus, the satellites are not due to periodic defects, but to polar domains [29]. Indeed the in-plane diffraction patterns are extremely similar to some of those observed around the 304 reflections for PTO films with vertical stripe domains in refs.[20, 21].

Stripes of alternate polarization form in a ferroelectric under open-circuit electrical boundary conditions in order to minimize the depolarizing field [20,30] and/or the epitaxial strain [10-12]. Since polarization is due to a shift of the $\mathrm{Pb}$ and $\mathrm{Ti}$ cations with respect to the oxygen cage, the different shift directions in the different domains produces contrast in the structure factors and causes the intensity modulations. Accordingly, there can only be structure-factor contrast (and therefore satellites) around those reciprocal lattice vectors that have at a)



b) ac phase

i) $\stackrel{c}{\quad \sim b}$

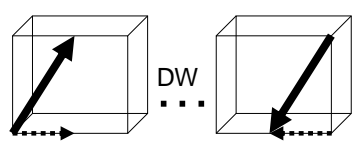

ii)

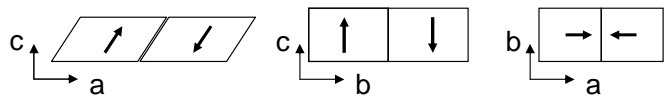

c) $r$ phase

i) $\quad c_{A} b$
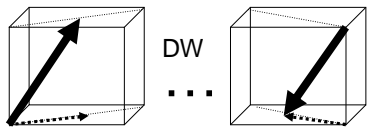

ii)

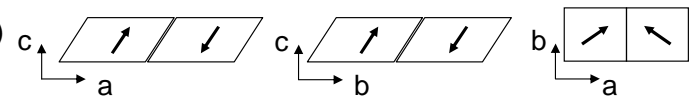

FIG. 4: a) Schematic representation of the observed reciprocal space maps and their implication for the polar domain orientation; Polar symmetry for b) ac-phase, and c) the $r$-phase. In b)and c): i) sketches the polar shifts in two unit cells at both sides of the domain wall and ii) represents the projections of the polarization in the $\{100\}$ pseudo-cubic planes for the proposed phases and domain configurations.

least one component parallel to the direction of the polar displacement[21]. Thus, the distribution of the satellites yields information about the domain geometry and size, and the Bragg peaks around which the satellites appear tell us about the direction of the polarization.

Therefore, from Figs. 1-3, we learn: i) Polarization must have both in-plane and out-of-plane components, since there are satellites around $(00 \mathrm{l}),(\mathrm{h} 0 \mathrm{l}),(\mathrm{h} 00),(0 \mathrm{k} 0)$ and $(\mathrm{hk} 0)$ reflections, all with the same size, and ii) the in-plane components of the polarization must be oriented head-to-head (and tail-to-tail) to produce modulation parallel to the in-plane reciprocal lattice vectors. In Figure 4 we have drawn a scheme of the domain orientations compatible with i) and ii). These are the $a c$ phase $\left(\mathrm{P}_{x} \neq\right.$ $0, \mathrm{P}_{y}=0, \mathrm{P}_{z} \neq 0$, monoclinic $\mathrm{Pm}$ ) with in-plane component of the polarization along the edges of the simple cubic cell (see Fig. 4b), and the $r$-phase $\left(\mathrm{P}_{x}=\mathrm{P}_{y} \neq \mathrm{P}_{z}\right.$, monoclinic space group $\mathrm{Cm}$ ) with in-plane component of the polarization along the face diagonals of the simple cubic cell (see Fig. 4c). To reproduce the observed maps in Fig. 4a, we consider four-fold in-plane symmetry and the existence of domain walls at 0 and $90^{\circ}$ in both phases. 
It is worth noting that the domain walls responsible for the in-plane satellites are charged, which is energetically costly. Although this is a surprising result, there are precedents of head-to-head polarized domains in other perovskites[31, 32]. If the polarization initially nucleates randomly, when domains with opposite polarization grow and encounter, whether or not they switch in order to match will depend on the balance between the energy needed for the switching and the amount of charge in the wall. If this charge is neutralized by defects, or directly short-circuited by the electrode, the domains will remain head-to-head. This picture is consistent with the bigger size of the domains in the films grown without SRO electrode. It is also worth noting that for ultra-thin films subjected to partially-screened electric fields, the polar orientation has been predicted to rotate between the bottom and the top surface in such a way that inplane head-to-head and tail-to-tail polar states appear at the top and the bottom layers of the film [12].

The out-of-plane lattice parameters are the same for the films grown directly on DSO as for those grown with an SRO layer. Therefore, the lower intensity of the satellites observed for the latter, specially around the outof-plane reflections (Figure 2-d) seems to indicate not a smaller out-of-plane polarization but rather a lack of modulation in the out-of-plane component of the polarization. This agrees with previous observations pointing that a lower electrode helps charge neutralization, thereby preventing the formation of c-axis antiparallel stripes [33]

In general our results agree with theoretical calculations that predict the rotation of the polarization, from perpendicular to parallel to the film, under tensile strain. However there is discrepancy between the phases we proposed and those predicted for $\mathrm{PbTiO}_{3}$ films. An $a a_{-}$ phase $\left(\mathrm{P}_{x}=\mathrm{P}_{y}, \mathrm{P}_{z}=0\right)$ have been theoretically predicted for the present strain state[7-9, 11], but it is incompatible with the observed $c$-axis polarization. Combinations of $a a$-phase and $c$-phase[8] or $a a$-phase and $r$-phase[34] have also been proposed, but they would imply two different out-out-plane lattice parameters, not observed here. The $r$-phase proposed by us has been predicted by Landau models for $\mathrm{PbTiO}_{3}$ under different (smaller) strain states[7], and by ab-initio calculations for PTO under hydrostatic pressure[35]. The $a c$ state has never been predicted for PTO. This discrepancy is in a sense not surprising, since most current theoretical calculations do not include electrical boundary conditions and are therefore not valid for very thin films. These boundary conditions have already been shown to affect the phase diagram of $\mathrm{BaTiO}_{3}[36]$ and the domain structure of $\mathrm{PbZr}_{1-x} \mathrm{Ti}_{x} \mathrm{O}_{3}$ under compressive strain [12].

In sum, fully coherent, very thin films of $\mathrm{PbTiO}_{3}$ under tensile strain display a modulation in reciprocal space indicating stripe domains with the polarization rotated away from the tetragonal axis. This new low-symmetry state may provide a route towards achieving large piezoelectric coefficients in undoped PTO [35].

Note added in proof: Several months after the measurements presented here, the stripe domains have disappeared due to charge neutralization. This confirms that their origin is polar and not crystallographic. The remarkably long stability of the stripes allowed their observation ex-situ in air, and confirms the good quality of the films [37].

We thank L. Bellaiche, T. Hibma, I. Kornev, J.F. Scott, A. Vlooswijk and V. Vonk for useful discussions and the Dutch organizations FOM and NWO for financial support. G.C. acknowledges financial support from the Marie-Curie Intra-European fellowship programme.
[1] M. Dawber et al., Rev. Mod. Phys. 77, 1083 (2005).

[2] G. A. Rossetti et al., Appl. Phys. Lett. 59, 2524 (1991).

[3] K. J. Choi et al., Science 306, 1005 (2004).

[4] J. H. Haeni et al., Nature (London) 430, 758 (2004).

[5] F. He et al., Phys. Rev. Lett. 94,176101 (2005).

[6] C. Basceri et al. , J. Appl. Phys. 82, 2497 (1997).

[7] N. A. Pertsev et al., Phys. Rev. Lett. 80, 1988 (1998).

[8] O. Diguez et al., Phys. Rev. B 72,144101 (2005).

[9] C. Bungaro and K. Rabe, Phys. Rev. B 69, 184101 (2004)

[10] J. S. Speck and W. Pompe, J. Appl. Phys. 76, 466 (1994); J.S. Speck et al., J. Appl. Phys. J. Appl. Phys. 78, 1696 (1995).

[11] Y.L. Li, L.Q. Chen, Appl. Phys. Lett. 88, 072905 (2006).

[12] I. Kornev et al., Phys. Rev. Lett. 93, 196104 (2004).

[13] B. S. Kwak et al. Phys. Rev. Lett. 68, 3733 (1992)

[14] S. Stemmer et al., Solid State Ionics 75, 43 (1995)

[15] M.D. Biegalski et al., J. Mat. Res. 20, 952 (2005).

[16] Although a small orthorhombic distortion was reported for bulk PTO below $-90^{\circ} \mathrm{C}$ (J. Kobayashi et al. , Phys. Rev. B 28, 3866 (1983)), the polar direction remains.

[17] R. Guo et al., Phys. Rev. Lett. 84, 5423 (2000).
[18] L. Bellaiche et al., Phys. Rev. Lett. 84, 5427 (2000).

[19] J. Junquera, P. Ghosez, Nature 422, 506 (2003).

[20] S. K. Streiffer et al., Phys. Rev. Lett. 89, 67601 (2002).

[21] D. Fong et al., Science 304, 1650 (2004).

[22] L. Despont et al., arXiv:cond-mat/0511084.

[23] A. Janssens et al., in preparation.

[24] G. Rijnders et al. Appl. Phys. Lett. 70, 1888 (1997)

[25] G. Shirane et al., Phys. Rev. 80, 1105 (1950).

[26] J.W. Matthews, A.E. Blakeslee, J. Cryst. Growth 27, 118 (1974).

[27] In some substrates we measured $\mathrm{a}=\mathrm{b}=3.950 \AA$ and in others $a=3.950 \AA, b=3.960 \AA$ (see Fig. $3 a$ ) and $b$ )). On the latter ones the PTO unit cell cannot not have the tetragonal symmetry of the bulk phase and is instead orthorhombic. The values measured by us differ slightly from those reported in the literature.

[28] F. He et al., Phys. Rev. B 70, 235405 (2004).

[29] Further evidence for a polar origin of the satellites was provided by their vanishing upon heating above $T_{C}$ in thicker (30nm) films (G. Catalan et al., in preparation).

[30] T. Mitsui, J. Furuichi, Phys. Rev. 90, 193 (1953). 
[31] E.A. Little, Phys. Rev. 98, 978 (1955).

[32] Y. Jin and W. Cao, Appl. Phys. Lett. 87, 7438 (2000); J. Han and W. Cao, Appl. Phys. Lett. 83, 2040 (2003).

[33] C. Thompson, communication, Intal. Meeting on Fundamental Physics of Ferroelectrics, Williamsburg 2004.

[34] N. A. Pertsev and V. G. Koukhar, Phys. Rev. Lett. 84,
$3722(2000)$.

[35] Z. Wu and R.E. Cohen, Phys. Rev. Lett. 95, 037601 (2005)

[36] Bo-Kuai Lai et al., Appl. Phys. Lett. 86, 132904 (2005).

[37] A. Kopal et al., Ferroelectrics 202, 267 (1997), 